\title{
Operator Splitting Method for Coupled Problems: Transport and Maxwell Equations
}

\author{
Jürgen Geiser \\ Department of Mathematics, Humboldt-Universität zu Berlin, Unter den Linden, Berlin, Germany \\ E-mail: geiser@mathematik.hu-berlin.de \\ Received March 31, 2011; revised April 13, 2011; accepted May 2, 2011
}

\begin{abstract}
In this article a new approach is considered for implementing operator splitting methods for transport problems, influenced by electric fields. Our motivation came to model PE-CVD (plasma-enhanced chemical vapor deposition) processes, means the flow of species to a gas-phase, which are influenced by an electric field. Such a field we can model by wave equations. The main contributions are to improve the standard discretization schemes of each part of the coupling equation. So we discuss an improvement with implicit RungeKutta methods instead of the Yee's algorithm. Further we balance the solver method between the Maxwell and Transport equation.
\end{abstract}

Keywords: Operator Splitting Method, Initial Value Problems, Iterative Solver Method, Stability Analysis, Beam Propagation Methods, Transport and Maxwell Equations

\section{Introduction}

We motivate our study by simulating thin film deposition processes that can be realized by PE-CVD (plasma enhanced chemical vapor deposition) processes, see $[1,2]$. For the deposition process, the influence of the electric fields to the transported gases in a plasma reactor is very important, see [3]. Therefore we deal with a simplified model of a coupled transport and Maxwell equations. While the transport equations modeled the transport of gaseous species and the Maxwell equation the influence of the underlying flow field.

We deal with the following equations

$$
\begin{gathered}
\partial_{t} u=-v_{x}\left(E_{z}(x, y)\right) \frac{\partial u}{\partial x}-v_{y} \frac{\partial u}{\partial y}+D \frac{\partial^{2} u}{\partial x^{2}} \\
+D \frac{\partial^{2} u}{\partial y^{2}}, \\
u\left(x, y, t_{0}\right)=u_{0}(x, y), \\
\frac{\partial H_{x}(x, y)}{\partial t}=-\frac{\partial E_{z}}{\partial y},(x, y, t) \in \Omega \times(0, T), \\
\frac{\partial H_{y}(x, y)}{\partial t}=\frac{\partial E_{z}}{\partial x},(x, y, t) \in \Omega \times(0, T),
\end{gathered}
$$

$$
\begin{aligned}
& \frac{\partial E_{z}(x, y)}{\partial t}=\frac{1}{\varepsilon}\left(\frac{\partial H_{y}}{\partial x}-\frac{\partial H_{x}}{\partial y}\right)-J_{\text {source }}, \\
& (x, y, t) \in \Omega \times(0, T)
\end{aligned}
$$

where $u$ is the concentration of the gaseous species, $E_{z}$ is the electric field and $H_{x}, H_{y}$ is the corresponding magnetic field in two dimensions. Further $v=\left(v_{x}, v_{y}\right)^{q}$ is the influenced velocity of the transport equation.

We concentrate on the numerical modeling and simulation of electrical fields, which are coupled with transport equations.

Several methods exist to solve electric field and are of interest.

One method for a stationary case of the electric field is a propagation method (BPM). This is a powerful tool to analyze linear and nonlinear light propagation in axially varying waveguides like directional couplers, tapered waveguides, S-shaped bent waveguides, and optical fibers [4-7]. The method has its origin in the field of propagation of electromagnetic beams in atmosphere, where the multi-physics modeling was done on the assumption that "the continuous gain medium may be approximated by a series of gain sheets with free propagation between the sheets" $[8,9]$. As it will be shown later on, this method is in fact a Strang-Marchuk operator splitting method $[10,11]$. Here we first describe the BPM [12]. We introduce the iterative splitting idea to couple 
Maxwell and Transport equations. Further a splitting analysis is presented. Numerical experiments are presented with respect to decoupled and coupled differential equations.

The paper is organized as follows. The discretization methods are described in Section 2. In Section 3, the applied operator splitting methods are presented. The error analysis of the coupled methods is studied in Section 4. The experiments of the new discretization methods and splitting methods are performed in Section 5. At the end of this paper we introduce future works.

\section{Discretization Method of the Maxwell Equation}

In the following we discuss the discretization methods for the Maxwell equation.

\subsection{FDTD Method: Yee’s Scheme}

Yee's scheme is the standard finite difference time-domain (FDTD) discretization of the following time dependent Maxwell curl equations

$$
\begin{aligned}
& -\mu_{0} \mu_{r} \frac{\partial \boldsymbol{H}}{\partial t}=\nabla \times \boldsymbol{E}, \\
& \varepsilon_{0} \varepsilon_{r} \frac{\partial \boldsymbol{E}}{\partial t}=\nabla \times \boldsymbol{H},
\end{aligned}
$$

where $\boldsymbol{E}=\left(E_{x}, E_{y}, E_{z}\right)(x, y, t)$ is the electric field, $\boldsymbol{H}=$ $\left(H_{x}, H_{y}, H_{z}\right)(x, y, t)$ is the magnetic field, $\varepsilon_{r}=\varepsilon(x, y)$ is the relative permittivity (given data), $\mu_{r}=1$ (nonmagnetic material) is the magnetic permeability. Here $\varepsilon_{0}, \mu_{0}$ are constants. It can be shown that if the divergence free conditions $\nabla \cdot\left(\varepsilon_{r} \boldsymbol{E}\right)=0$ and $\nabla \cdot(\boldsymbol{H})=0$ are satisfied at $t=0$, then they are satisfied for all time. This is the case for our setting. Therefore it is enough to consider only the above curl equation. Rewriting them component-wise, we get in our case

$$
\begin{gathered}
-\mu_{0} \frac{\partial H_{x}}{\partial t}=\frac{\partial E_{z}}{\partial y}-\frac{\partial E_{y}}{\partial z} \\
-\mu_{0} \frac{\partial H_{y}}{\partial t}=\frac{\partial E_{x}}{\partial z}-\frac{\partial E_{z}}{\partial x} \\
\varepsilon_{0} \varepsilon_{r} \frac{\partial E_{z}}{\partial t}=\frac{\partial H_{y}}{\partial x}-\frac{\partial H_{x}}{\partial y}
\end{gathered}
$$

Let $\Delta x, \Delta y$ are spatial discretizations, and $\Delta t$ is a time step. We use the following notation

$$
F^{n}(i, j)=F(i \Delta x, j \Delta y, n \Delta t) .
$$

Let $\alpha$ represents a spatial coordinate such as $x, y$. The goal of Yee's scheme is to compute the approxima- tions for the various components $E_{\alpha}$ of $\boldsymbol{E}$ and $H_{\alpha}$ of $\boldsymbol{H}$ at the following spatial locations and temporal instants:

$$
\begin{aligned}
& E_{\alpha}=\left\{\begin{array}{l}
\text { spatial coordinate } \alpha \text { :half integer } \\
\text { other spatial coordinate }: \text { integer } \\
\text { time }: \text { integer }
\end{array}\right. \\
& H_{\alpha}=\left\{\begin{array}{l}
\text { spatial coordinate } \alpha \text { :integer } \\
\text { other spatial coordinate }: \text { half integer } \\
\text { time }: \text { half integer }
\end{array}\right.
\end{aligned}
$$

Thus the distributions/grid of various components are staggered in space and in time. This is one of the two unique characteristics of the Yee's scheme. The second unique characteristic is that the various spatial derivatives in Equations (8) - (10) are computed across the one spatial cell, i.e. the difference center for the central difference approximation of the spatial derivative is the mid point of one cell length in the corresponding direction of the derivative. Thus the Yee's scheme approximates Equations (8) - (10) at the following points:

$$
\begin{gathered}
\text { Equation }(8) \mapsto(i \Delta x,(j+1 / 2) \Delta y, n \Delta t) \\
\text { Equation }(9) \mapsto((i+1 / 2) \Delta x, j \Delta y, n \Delta t) \\
\text { Equation }(10) \mapsto(i \Delta x, j \Delta y,(n+1 / 2) \Delta t)
\end{gathered}
$$

Such a staggered uncollocated arrangement gives the Yee's scheme several nice numerical and physical properties, see [13]. Then we get finite-difference approximations as:

$$
\begin{aligned}
& H_{x}^{n+\frac{1}{2}}\left(i, j+\frac{1}{2}\right) \\
= & H_{x}^{n-\frac{1}{2}}\left(i, j+\frac{1}{2}\right)-\frac{1}{\mu_{0}} \frac{\Delta t}{\Delta y}\left[E_{z}^{n}(i, j+1)-E_{z}^{n}(i, j)\right], \\
& H_{y}^{n+\frac{1}{2}}\left(i+\frac{1}{2}, j\right) \\
= & H_{y}^{n-\frac{1}{2}}\left(i+\frac{1}{2}, j\right)+\frac{1}{\mu_{0}} \frac{\Delta t}{\Delta x}\left[E_{z}^{n}(i+1, j)-E_{z}^{n}(i, j)\right], \\
& E_{z}^{n+1}(i, j) \\
= & E_{z}^{n}(i, j) \\
+ & \frac{1}{\varepsilon_{0} \varepsilon_{r}} \frac{\Delta x}{\Delta x}\left[H_{y}^{n+\frac{1}{2}}\left(i+\frac{1}{2}, j\right)-H_{y}^{n+\frac{1}{2}}\left(i-\frac{1}{2}, j\right)\right] \\
& \frac{1}{\varepsilon_{0} \varepsilon_{r}} \frac{\Delta t}{\Delta y}\left[H_{x}^{n+\frac{1}{2}}\left(i, j+\frac{1}{2}\right)-H_{x}^{n+\frac{1}{2}}\left(i, j-\frac{1}{2}\right)\right] .
\end{aligned}
$$


In Equation (19) the relative permittivity $\varepsilon_{r}$ is computed at the corresponding difference center as given by Equation (16). At the interface between two media, $\varepsilon_{r}$ is approximated by the average value.

Conditions for the Yee's algorithm:

- The CFL stability condition for the Yee's FDTD method is

$$
\Delta t \leq \frac{1}{c} \sqrt{\frac{1}{(\Delta x)^{2}}+\frac{1}{(\Delta y)^{2}}}
$$

where $c$ is the speed of light in vacuum, see [13].

- To restrict the unbounded domain to finite domain, one uses absorbing boundary condition like the perfectly matched layers, see [14,15].

Remark 1. Often for more accurate problems a Yee's algorithm which is second order in time and second order in space is often to low. For higher order methods in time and space can be constructed but are often to delicate and expensive to implement, see [16,17]. We propose to improve with higher order implicit Runge-Kutta methods with an idea to sparse matrices schemes, which saves additional memory.

\subsection{Improved Time Discretization Methods for Maxwell Equation}

Based on the problem of reconstructing a higher order Yee's algorithm, we deal with separate improvement of the discretization schemes.

While the spatial discretization of the Yee's algorithm is a second order difference scheme, the time discretization is also only a second order scheme.

Here we see the deficits of only improving the spatial scheme with higher order schemes and leave the timediscretization with a second order scheme.

We propose an improved time-discretization scheme of higher order and apply fine spatial grids, while the time error is at least larger, see [18].

We deal with higher order time-discretization methods. Therefore we propose the Runge-Kutta as adapted timediscretization methods to reach higher order results. For the time-discretization we use the following higher order discretization methods.

We deal with the following semi-discretized partial differential equations, such equations are used in each iterative splitting step:

$$
\begin{aligned}
\frac{\partial u}{\partial t} & =A u+f(t), \\
u_{n} & =u\left(t^{n}\right),
\end{aligned}
$$

where $A$ is the operator that we implicit solve in the equation and $f(t)=B \tilde{u}(t)$ is the explicit operator, with a previous solution $\tilde{u}$, e.g. last iterative solution.

\subsubsection{Higher Order Time-Discretization Methods with Runge-Kutta Methods}

We deal with the following Maxwell equation, given as:

$$
\begin{aligned}
\frac{\partial E_{z}}{\partial t} & =\frac{1}{\varepsilon}\left[\frac{\partial H_{y}}{\partial x}-\frac{\partial H_{x}}{\partial y}\right]-J \\
& =\mathrm{I}\left(H_{x}, H_{y}, J\right)=\frac{1}{\varepsilon}\left[B_{1} H_{y}-B_{2} H_{x}\right]-J \\
\frac{\partial H_{x}}{\partial t} & =-\frac{1}{\mu} \frac{\partial E_{z}}{\partial y}=\mathrm{II}\left(E_{z}\right)=-\frac{1}{\mu} C_{1} E_{z} \\
\frac{\partial H_{y}}{\partial t} & =\frac{1}{\mu} \frac{\partial E_{z}}{\partial x}=\mathrm{III}\left(E_{z}\right)=\frac{1}{\mu} C_{2} E_{z}
\end{aligned}
$$

For the boundary conditions we assume periodic boundary conditions. That means we use the identification

$$
\begin{gathered}
E_{z}(N, i)=E_{z}(1, i) \\
E_{z}(i, N)=E_{z}(i, 1) \forall i=1, \cdots, N .
\end{gathered}
$$

Remark 2. For the stationary field, we apply a periodic boundary condition, which is sufficient. The Mur absorbing boundary condition, see [5], is used for the instationary field, while respecting the influence of the changes at the boundaries.

To get a first realization of an open boundary in the case of the line-source we use symmetry and a combination of PBC and Mur's first order ABC. For the boundarys orthogonal to the propagation direction of the field (left-right) it is useful to work with Mur's ABC.

\subsubsection{Mur's ABC}

We can interpret the electromagnetical field as a wave that has to fulfill the homogeneous wave equation.

$$
\begin{aligned}
\square \Phi=0 \Leftrightarrow & \left(\Delta-\frac{1}{c^{2}} \frac{\partial^{2}}{\partial t^{2}}\right) \Phi=0\left[\frac{1}{c^{2}}=\mu \varepsilon=\mu_{0} \mu_{r} \varepsilon_{0} \varepsilon_{r}\right] \\
\Leftrightarrow & \left(\frac{\partial^{2}}{\partial x^{2}}+\frac{\partial^{2}}{\partial y^{2}}-\frac{1}{c^{2}} \frac{\partial^{2}}{\partial t^{2}}\right) \Phi=0 \\
\Leftrightarrow & \left(D_{x}^{2}+D_{y}^{2}-\frac{1}{c^{2}} D_{t}^{2}\right) \Phi=0 \\
\Leftrightarrow & \left(D_{x}-\frac{1}{c} D_{t} \sqrt{1-\frac{D_{y}^{2} \cdot c^{2}}{D_{t}^{2}}}\right) \\
& \times\left(D_{x}+\frac{1}{c} D_{t} \sqrt{1-\frac{D_{y}^{2} \cdot c^{2}}{D_{t}^{2}}}\right) \Phi=0
\end{aligned}
$$




$$
\begin{aligned}
& \Leftrightarrow(D_{x}-\frac{1}{c} D_{t} \underbrace{\sqrt{1-V^{2}}}_{\left(^{*}\right)})\left(D_{x}+\frac{1}{c} D_{t} \sqrt{1-V^{2}}\right) \Phi=0 \\
& \Leftrightarrow \square_{x}^{-} \square_{x}^{+} \Phi=0
\end{aligned}
$$

Waves that satisfy $\square_{x}^{-} \Phi=0$ only propagate in $-x$-direction and those that satisfy $\square_{x}^{+} \Phi=0$ only propagate in $+x$-direction. An analogous formulation can be given for the $-y$ and $y$ direction.

To handle $(*)$ it is comfortable to do a Taylor expansion around 0 .

$$
\begin{aligned}
\sqrt{1-V^{2}}= & 1-\frac{V}{\sqrt{1-V^{2}}}(0) \cdot V-\frac{1}{2\left(1-V^{2}\right)^{\frac{3}{2}}}(0) \cdot V^{2} \\
& -\frac{V}{\left(1-V^{2}\right)^{\frac{5}{2}}}(0) \cdot V^{3}+O\left(V^{4}\right) \\
= & 1-\frac{1}{2} V^{2}+O\left(V^{4}\right) \\
= & 1+O\left(V^{2}\right)
\end{aligned}
$$

Considering (36) equation (32) turns to

$$
\left(\frac{\partial}{\partial x}-\frac{1}{c} \frac{\partial}{\partial t}\right)\left(\frac{\partial}{\partial x}+\frac{1}{c} \frac{\partial}{\partial t}\right) \Phi=0,
$$

which is Mur's ABC with first order accuracy. As a first attempt to model an open boundary we will use this.

Left boundary $\left(x=x_{0}\right)$

For the left boundary we have do discretize the following equation:

$$
\frac{\partial \Phi}{\partial x}=\frac{1}{c} \frac{\partial \Phi}{\partial t} .
$$

This can be done with a FDM-scheme as follows.

$$
\begin{aligned}
& \left.\frac{\partial \Phi}{\partial x}\right|_{x=x_{0}+\frac{1}{2} \Delta x}=\frac{1}{\Delta x}\left(\Phi^{n+\frac{1}{2}}(j, 2)-\Phi^{n+\frac{1}{2}}(j, 1)\right) ; \\
& \left.\frac{\partial \Phi}{\partial t}\right|_{t=t} ^{n+\frac{1}{2}}=\frac{1}{\Delta t}\left(\Phi^{n+1}\left(j, \frac{3}{2}\right)-\Phi^{n}\left(j, \frac{3}{2}\right)\right)
\end{aligned}
$$

with

$$
\begin{aligned}
& \Phi^{n+\frac{1}{2}}(j, 2)=\frac{1}{2}\left(\Phi^{n+1}(j, 2)+\Phi^{n}(j, 2)\right) ; \\
& \Phi^{n+\frac{1}{2}}(j, 1)=\frac{1}{2}\left(\Phi^{n+1}(j, 1)+\Phi^{n}(j, 1)\right)
\end{aligned}
$$

and

$$
\begin{aligned}
& \Phi^{n+1}\left(j, \frac{3}{2}\right)=\frac{1}{2}\left(\Phi^{n+1}(j, 2)+\Phi^{n+1}(j, 1)\right) ; \\
& \Phi^{n}\left(j, \frac{3}{2}\right)=\frac{1}{2}\left(\Phi^{n}(j, 2)+\Phi^{n}(j, 1)\right)
\end{aligned}
$$

this leads to

$$
\Phi^{n+1}(j, 1)=\Phi^{n}(j, 2)+\frac{c \Delta t-\Delta x}{c \Delta t+\Delta x}\left(\Phi^{n+1}(j, 2)-\Phi^{n}(j, 1)\right)
$$

It is easy to see that this tool does not satisfy completely because it only has first order accuracy and even more important it only absorbs the part of the wave that propagates orthogonal to the boundary.

But there are also a few advantages. Mur's ABC has to be applied only to the $E_{z}$ field because $H_{x}$ and $H_{y}$ are dealt with automatically through the ordinary updatestep. The second advantage of Mur's ABC is the low numeric expense.

For the boundaries parallel to the propagation direction (top and bottom) we use the PBC. The symmetry of our setting garanties that the inflow and the outflow of the field equalize each other.

But with the eye on the next simulations with less symmetry it seams to be necessary to use perfectly matched layers.

These 3 equations above mark the starting point. The spatial part of each equation is discretised and is calculated with the help of the matrix-operators $B_{1}, B_{2}, C_{1}, C_{2}$ (centered differences corresponding to the 2 dimensional Yee-lattrice).

In the following we are using the general Butcher-table for (3-stage) Runge-Kutta-methods to get a clear notation.

$$
\begin{array}{l|lll}
c_{1} & a_{11} & a_{12} & a_{13} \\
c_{2} & a_{21} & a_{22} & a_{23} \\
c_{3} & a_{31} & a_{32} & a_{33} \\
\hline & b_{1} & b_{2} & b_{3}
\end{array}=\frac{c}{b^{T}}
$$

Let $\Delta t$ denote the stepping time and $t_{j}^{(i)}=t^{(i)}+\Delta t c_{j}$. The step from $t^{(i)}$ to $t^{(i+1)}$ in (23) - (25) can now be written in the following way.

$$
\begin{aligned}
& E_{z}^{(i+1)}=E_{z}^{(i)}+\Delta t\left(b_{1} k_{1}^{\mathrm{I}}+b_{2} k_{2}^{\mathrm{I}}+b_{3} k_{3}^{\mathrm{I}}\right) \\
& H_{x}^{(i+1)}=H_{x}^{(i)}+\Delta t\left(b_{1} k_{1}^{\mathrm{II}}+b_{2} k_{2}^{\mathrm{II}}+b_{3} k_{3}^{\mathrm{II}}\right) \\
& H_{y}^{(i+1)}=H_{y}^{(i)}+\Delta t\left(b_{1} k_{1}^{\mathrm{III}}+b_{2} k_{2}^{\mathrm{III}}+b_{3} k_{3}^{\mathrm{III}}\right)
\end{aligned}
$$

where $k_{j}^{M}=M\left(H_{x_{i}}^{(i)}, H_{y_{j}}^{(i)}, E_{z_{j}}^{(i)}, J_{j}^{(i)}\right)$ for $M \in\{$ I, II, III $\}$ and $j \in\{1,2,3\}$. With

$$
\begin{aligned}
& E_{z_{j}}^{(i)}=E_{z}\left(t_{j}^{(i)}\right)=E_{z}^{(i)}+\Delta t\left(\sum_{l=1}^{3} a_{j l} k_{l}^{\mathrm{I}}\right) \\
& H_{x_{j}}^{(i)}=H_{x}\left(t_{j}^{(i)}\right)=H_{x}^{(i)}+\Delta t\left(\sum_{l=1}^{3} a_{j l} k_{l}^{\mathrm{II}}\right) \\
& H_{y_{j}}^{(i)}=H_{y}\left(t_{j}^{(i)}\right)=H_{y}^{(i)}+\Delta t\left(\sum_{l=1}^{3} a_{j l} k_{l}^{\mathrm{III}}\right)
\end{aligned}
$$




$$
J_{j}^{(i)}=J\left(t_{j}^{(i)}\right) \text { which is known (in our case) }
$$

For a better legibility and because the focused point of time does not change, we write $\left(E_{z}^{j}, H_{x}^{j}, H_{y}^{j}, J^{j}\right)$ instead of $\left(E_{z_{j}}^{(i)}, H_{x_{j}}^{(i)}, H_{y_{j}}^{(i)}, J_{j}^{(i)}\right)$.

Combining (23) - (25) and (47) - (50) give 9 equations

$$
\begin{aligned}
& E_{z}^{j}=E_{z}+\Delta t\left\{\sum_{l=1}^{3} a_{j} l\left[\frac{1}{\varepsilon}\left(B_{1} H_{y}^{l}-B_{2} H_{x}^{l}\right)-J^{l}\right]\right\} \\
& H_{x}^{j}=H_{x}-\Delta t\left(\sum_{l=1}^{3} a_{j} l \frac{1}{\mu} C_{1} E_{z}^{l}\right) \\
& H_{y}^{j}=H_{y}+\Delta t\left(\sum_{l=1}^{3} a_{j} l \frac{1}{\mu} C_{2} E_{z}^{l}\right) \quad j \in\{1,2,3\}
\end{aligned}
$$

Remark 3. $1 / \varepsilon$ and $1 / \mu$ are also realized as matrices, such that $1 / \varepsilon=1 / \varepsilon(x, y)$ and $1 / \mu=1 / \mu(x, y)$.

Remark 4. The scheme above is only correct for isotropic media because in the not isotropic case it is necessary to consider $\mu=\left(\mu_{x}, \mu_{y}\right)$.

Taking the 6 equations of (52) and (53) and putting them together with the 3 equations of (51) leads to the following linear equation system which needs to be solved.

$$
\begin{aligned}
& \left(\begin{array}{l}
Q^{1}-E_{z} \\
Q^{2}-E_{z} \\
Q^{3}-E_{z}
\end{array}\right) \\
& =\left(\begin{array}{lll}
\left\langle R_{1}^{A}, C_{1}^{A}\right\rangle S-I & \left\langle R_{1}^{A}, C_{2}^{A}\right\rangle S & \left\langle R_{1}^{A}, C_{3}^{A}\right\rangle S \\
\left\langle R_{2}^{A}, C_{1}^{A}\right\rangle S & \left\langle R_{2}^{A}, C_{2}^{A}\right\rangle S-I & \left\langle R_{2}^{A}, C_{3}^{A}\right\rangle S \\
\left\langle R_{3}^{A}, C_{1}^{A}\right\rangle S & \left\langle R_{3}^{A}, C_{2}^{A}\right\rangle S & \left\langle R_{3}^{A}, C_{3}^{A}\right\rangle S-I
\end{array}\right) \\
& \left(\begin{array}{c}
E_{z}^{1} \\
E_{z}^{2} \\
E_{z}^{3}
\end{array}\right) \\
& \left(\begin{array}{l}
Q^{1}-E_{z} \\
Q^{2}-E_{z} \\
Q^{3}-E_{z}
\end{array}\right)=\left(\begin{array}{lll}
a_{11}^{2} S-I & a_{12}^{2} S & a_{13}^{2} S \\
a_{21}^{2} S & a_{22}^{2} S-I & a_{23}^{2} S \\
a_{31}^{2} S & a_{32}^{2} S & a_{33}^{2} S-I
\end{array}\right) \cdot\left(\begin{array}{c}
E_{z}^{1} \\
E_{z}^{2} \\
E_{z}^{3}
\end{array}\right)(55) \\
& \left(\begin{array}{l}
Q^{1}-E_{z} \\
Q^{2}-E_{z} \\
Q^{3}-E_{z}
\end{array}\right)=\left[\mathcal{A}^{2} \cdot\left(\begin{array}{ccc}
S & 0 & 0 \\
0 & S & 0 \\
0 & 0 & S
\end{array}\right)-\left(\begin{array}{ccc}
I & 0 & 0 \\
0 & I & 0 \\
0 & 0 & I
\end{array}\right)\right]\left(\begin{array}{c}
E_{z}^{1} \\
E_{z}^{2} \\
E_{z}^{3}
\end{array}\right) \\
& Q^{j}:=\Delta t\left[\left\langle R_{j}^{A}, 1\right\rangle \frac{1}{\varepsilon}\left(B_{1} H_{y}-B_{2} H_{x}\right)+\left\langle R_{j}^{A}, J\right\rangle\right] \\
& R_{j}^{A}:=j \text {-th row of } A \\
& C_{j}^{A}:=j \text {-th column of } A
\end{aligned}
$$

$$
\begin{aligned}
& 1:=(1,1,1) \\
& J:=\left(J^{1}, J^{2}, J^{3}\right) \\
& S:=\Delta t^{2} \frac{1}{\varepsilon} \frac{1}{\mu}\left(B_{1} C_{2}-B_{2} C_{1}\right) \\
& a_{i j}^{2}:=\left(A^{2}\right)_{i j}=\left\langle R_{i}^{A}, C_{j}^{A}\right\rangle \\
& \mathcal{A}^{2}:=\left(\begin{array}{lll}
\mathbf{a}_{11}^{2} & \mathbf{a}_{12}^{2} & \mathbf{a}_{13}^{2} \\
\mathbf{a}_{21}^{2} & \mathbf{a}_{22}^{2} & \mathbf{a}_{23}^{2} \\
\mathbf{a}_{31}^{2} & \mathbf{a}_{32}^{2} & \mathbf{a}_{33}^{2}
\end{array}\right) \\
& \mathbf{a}_{i j}^{2}=\underbrace{\left(\begin{array}{lll}
a_{i j}^{2} & & 0 \\
& \ddots & \\
0 & & a_{i j}^{2}
\end{array}\right)}_{n \cdot m \text { times }}\} n \cdot m \text { times } \\
& I:=\operatorname{Identity}(n \cdot m)
\end{aligned}
$$

where $a_{i j}, b_{i}$ and $c_{j}$ are the Runge-Kutta coefficients.

To be more precisely: If we have $n \times m$ points in our region, there are $3 \cdot n \cdot m$ equations to solve.

With this result we are able to calculate (52) and (53). So that it is finally possible to do the step ((44) - (46)).

Remark 5. For an optimization of the time-discretization scheme, we can neglect some of the outerdiagonals of the RK methods, which leads to SDIRK methods. We have the benefit in faster computations, without reducing the accuracy. For higher time-discretizations we have to taken into account also higher spatial discretization scheme.

\subsubsection{Stability Analysis of the Implicit Discretizations} We deal with the following discretized equation systems:

$$
E_{z, i}^{n+1}=(I-\tilde{A})^{-1}\left(E_{z}^{n}+\tilde{C}_{1} H_{y}^{n}+\tilde{C}_{2} H_{x}^{n}-J\left(u^{n}\right)-J\left(u_{i-1}^{n+1}\right)\right)
$$

where $i$ is the iteration index of the coupling scheme.

Definition 1. We have a positive definite matrix $M$ $\left(n \times n\right.$ real symmetric matrix), if $z^{\mathrm{T}} A z>0$ for all non-zero vectors $z$ with real entries $\left(z \in \mathbb{R}^{n}\right)$, where $z^{T}$ denotes the transpose of $z$.

Example 1. For finite difference discretization, e.g. $(1 / \Delta x)[-12-1]$, it is sufficient to show, that the sum of the outer-diagonals are equal or less than the diagonal.

$\sum_{j=1}^{n} a_{i, j} \leq a_{i i}$ for $i=1, \cdots, n$ and $n$ is the number of discretization points.

We have the following assumptions:

\section{Assumption 1.}

1) We assume $\tilde{A}$ is positive definite, and therefore we have 


$$
\left\|(I-\tilde{A})^{-1}\right\| \leq 1
$$

see [19].

2) We assume

$$
\left\|\left(E_{z}^{n}+\tilde{C}_{1} H_{y}^{n}+\tilde{C}_{2} H_{x}^{n}-J\left(u^{n}\right)-J\left(u_{i-1}^{n+1}\right)\right)\right\| \leq\left\|E_{z}^{n}\right\|
$$

The stability is given with in the following Theorem:

Theorem 1. Given is the numerical scheme (70) and we have the assumptions 1 .

The scheme is stable for all iterative steps $i$.

Proof 1. Based on the assumptions we can bound the inverse matrix, also the previous solution is bounded. Then scheme is stable.

We have the following proof idea:

Based on the assumption $1, \tilde{A}$ is positive definite and the estimation of the remaining term, we have :

$$
E_{z, i}^{n+1} \leq \|\left(E_{z}^{n} \| .\right.
$$

So we have an upper bound of the iterative results, given by the previous solution at time $t^{n}$.

\subsection{Discretization Methods of the Convection-Diffusion Equation}

For the 3 dimensional convection-diffusion equation we apply a second order finite difference scheme in space and a higher order discretization scheme in time.

$$
\begin{aligned}
& \frac{\partial u}{\partial t}=-v \nabla u+D \Delta u, \\
&=-v_{x} \frac{\partial u}{\partial x}-v_{y} \frac{\partial u}{\partial y}-v_{z} \frac{\partial u}{\partial z}+D \frac{\partial^{2} u}{\partial x^{2}} \\
&+D \frac{\partial^{2} u}{\partial y^{2}}+D \frac{\partial^{2} u}{\partial z^{2}}, \\
& u\left(x, t_{0}\right)=u_{0}(x),
\end{aligned}
$$

We apply dimensional splitting to our problem

$$
\frac{\partial u}{\partial t}=A_{x} u+A_{y} u+A_{z} u
$$

where

$$
A_{x}=-v_{x} \frac{\partial u}{\partial x}+D \frac{\partial^{2} u}{\partial x^{2}} .
$$

We use a 1st order upwind scheme for $\partial / \partial x$ and a 2nd order central difference scheme for $\partial^{2} / \partial x^{2}$. By introducing the artificial diffusion constant $D_{x}=D-$ $\left(v_{x} \Delta x\right) / 2$ we achieve a 2 nd order finite difference scheme

$$
\begin{aligned}
L_{x} u(x) & =-v_{x} \frac{u(x)-u(x-\Delta x)}{\Delta x} \\
& +D_{x} \frac{u(x+\Delta x)-2 u(x)+u(x-\Delta x)}{\Delta x^{2}} .
\end{aligned}
$$

because the new diffusion constant eliminates the first order error (i.e. the numerical viscosity) of the Taylor expansion of the upwind scheme. $L_{y} u$ and $L_{z} u$ are derived in the same way.

For the discretization in time we use several explicit Runge-Kutta and Adam-Bashforth methods, this leads to restrictions of the step-size in time but on the other hand the cost of implicit methods is much to high in this 3-dimensional case.

\subsubsection{Adam-Bashforth Methods}

$$
\begin{aligned}
y_{n+1} & =y_{n}+h \sum_{j=0}^{s} b_{j} f\left(t_{n-j}, y_{n-j}\right) \\
b_{j} & =\frac{(-1)^{j}}{j !(s-j) !} \int_{0_{i=0, i \neq j}^{1}}^{s}(u+i) \mathrm{d} u, \quad j=0, \cdots, s .
\end{aligned}
$$

We consider here

$s=1$ (first order)

$$
y_{n+1}=y_{n}+h\left(\frac{3}{2} f\left(t_{n}, y_{n}\right)-\frac{1}{2} f\left(t_{n-1}, y_{n-1}\right)\right)
$$

and

$s=2$ (second order)

$$
\begin{aligned}
y_{n+1}= & y_{n}+h\left(\frac{23}{12} f\left(t_{n}, y_{n}\right)-\frac{16}{12} f\left(t_{n-1}, y_{n-1}\right)\right. \\
& \left.+\frac{5}{12} f\left(t_{n-2}, y_{n-2}\right)\right)
\end{aligned}
$$

\subsubsection{Explicit Runge-Kutta Methods}

In general a s-stage Runge-Kutta method can be written in the following way:

$$
y_{n+1}=y_{n}+h \sum_{j=1}^{s} b_{j} k_{j}
$$

where

$$
k_{j}=f\left(t_{n}+h c_{j}, y_{n}+h \sum_{l=1}^{s} a_{j l} k_{l}\right)
$$

We will take into account the following two:

Heun's third-order

$$
\begin{array}{l|lll}
0 & 0 & 0 & 0 \\
\frac{1}{3} & \frac{1}{3} & 0 & 0 \\
\frac{2}{3} & 0 & \frac{2}{3} & 0
\end{array}=\frac{c}{\mid b^{T}}
$$

and

Kutta's classical fourth-order 


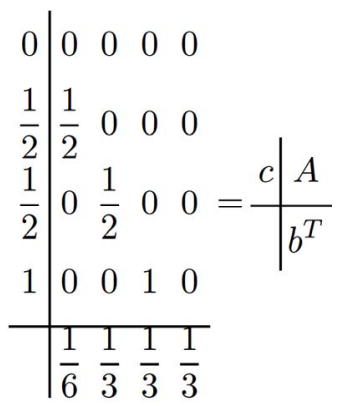

\section{Splitting Methods to Couple Maxwell and Convection Diffusion Equation}

We concentrate on the splitting methods, which can be classified as classical and iterative splitting methods.

We propose iterative splitting methods by discussing the additive iterative splitting methods, see [20,21].

We consider the following the linear problem

$$
\partial_{t} c(t)=A c(t)+B c(t)
$$

where the initial conditions are $c^{n}=c\left(t^{n}\right)$. The operators $A$ and $B$ are spatially discretized operators, e.g. they correspond in space to the discretized convection and diffusion operators (matrices). Hence, they can be considered as bounded operators.

\section{Iterative Splitting Methods}

The following algorithm is based on the iteration with fixed splitting discretization step size $\tau$. On the time interval $\left[t^{n}, t^{n+1}\right]$ we solve the following subproblems consecutively for $i=1,3, \cdots, 2 m+1$, cf. [20,21].

$$
\begin{aligned}
& \frac{\partial c_{i}(t)}{\partial t}=A c_{i}(t)+B c_{i-1}(t), \text { with } c_{i}\left(t^{n}\right)=c^{n}, \\
& \frac{\partial c_{i+1}(t)}{\partial t}=A c_{i}(t)+B c_{i+1}(t), \text { with } c_{i+1}\left(t^{n}\right)=c^{n},
\end{aligned}
$$

where $c_{0} \equiv 0$ and $c^{n}$ is the known split approximation at time level $t=t^{n}$. The split approximation at time level $t=t^{n+1}$ is defined as $c^{n+1}=c_{2 m+2}\left(t^{n+1}\right)$. (Clearly, the function $c_{i+1}(t)$ depends on the interval $\left[t^{n}, t^{n+1}\right]$, too, but for the sake of simplicity, in our notation we omit the dependence on $n$ ).

In the following we analyze the convergence and the rate of the convergence of the method (80) - (81) for $m$ tending to infinity for the linear operators $A, B: X \rightarrow X$, where we assume that these operators and their sum are generators of the $C_{0}$ semigroups. We emphasize that these operators aren't necessarily bounded, thus the convergence is examined in a general Banach space setting.

Theorem 2. Let us consider the abstract Cauchy problem in a Banach space $\mathbf{X}$

$$
\begin{gathered}
\partial_{t} c(t)=A c(t)+B c(t), \quad 0<t \leq T, \\
c(0)=c_{0},
\end{gathered}
$$

where $A, B, A+B: X \rightarrow X$ are given linear operators being generators of the $C_{0}$ semigroup and $c_{0} \in X$ is a given element. Then the iteration process (80) - (81) is convergent and the rate of the convergence is of higher order.

The proof can be found in [22].

Remark 6. When $A$ and $B$ are matrices (i.e. (80) - (81) is a system of ordinary differential equations), for the growth estimation we can use the concept of the logarithmic norm, see e.g. [23]. Hence, for many important classes of matrices we can prove the validity.

Remark 7. We note that a huge class of important differential operators generate a contractive semigroup. This means that for such problems-assuming the exact solvability of the split subproblems-the iterative splitting method converges in higher order to the exact solution.

In the next subsection we present the used time-discretization methods.

\section{Error Analysis: Coupling Methods}

For the coupling methods we deal with nonlinear differential equations of the following type:

$$
\frac{\mathrm{d} c}{\mathrm{~d} t}=A(c(t)) c(t)+B(c(t)) c(t), \text { with } c\left(t^{n}\right)=c^{n},
$$

where $c=\left(H_{x}, H_{y}, E_{z}, u\right)$, with $H_{x}, H_{y}$ is the magnetic field, $E_{z}$ is the electric field and $u$ is the concentration of the species.

The main idea is to bound the operators $A(c(t))$ and $B(c(t))$ in the discretized equation to satisfy a stable method.

A first idea is the fix-point scheme, that is discussed in the following subsection.

\section{Iterative Operator-Splitting Method as a Fix-Point Scheme}

The iterative operator-splitting method is used as a fix-point scheme to linearize the nonlinear operators, see $[21,24]$.

We restrict our attention to time-dependent partial differential equations of the form:

$$
\frac{\mathrm{d} u}{\mathrm{~d} t}=A(u(t)) u(t)+B(u(t)) u(t), \text { with } u\left(t^{n}\right)=c^{n},
$$

where $A(u), B(u): X \rightarrow X$ are linear and densely defined in the real Banach space $X$, involving only spatial derivatives of $c$, see [25]. In the following we discuss the standard iterative operator-splitting methods as a fixpoint iteration method to linearize the operators. 
We split our nonlinear differential equation (84) by applying:

$$
\begin{aligned}
& \frac{\mathrm{d} u_{i}(t)}{d t}=A\left(u_{i-1}(t)\right) u_{i}(t)+B\left(u_{i-1}(t)\right) u_{i-1}(t), \\
& \text { with } u_{i}\left(t^{n}\right)=c^{n}, \\
& \frac{\mathrm{d} u_{i+1}(t)}{d t}=A\left(u_{i-1}(t)\right) u_{i}(t)+B\left(u_{i-1}(t)\right) u_{i+1}, \\
& \text { with } u_{i+1}\left(t^{n}\right)=c^{n},
\end{aligned}
$$

where the time step is $\tau=t^{n+1}-t^{n}$. The iterations are $i=1,3, \cdots, 2 m+1 . \quad u_{0}(t)=c_{n}$ is the starting solution, where we assume the solution $c^{n+1}$ is near $c^{n}$, or $u_{0}(t)=0$. So we have to solve the local fix-point problem. $c^{n}$ is the known split approximation at the time level $t=t^{n}$.

The split approximation at time level $t=t^{n+1}$ is defined as $c^{n+1}=u_{2 m+2}\left(t^{n+1}\right)$. We assume the operators $A\left(u_{i-1}\right), B\left(u_{i-1}\right): X \rightarrow X$ to be linear and densely defined on the real Banach space $X$, for $i=1,3, \cdots, 2 m+1$.

Here the linearization is done with respect to the iterations, such that $A\left(u_{i-1}\right), B\left(u_{i-1}\right)$ are at least non-dependent operators in the iterative equations, and we can apply the linear theory.

The linearization is at least in the first equation $A\left(u_{i-1}\right) \approx A\left(u_{i}\right)$, and in the second equation $B\left(u_{i-1}\right) \approx$ $B\left(u_{i+1}\right)$

We have

$$
\left\|A\left(u_{i-1}\left(t^{n+1}\right)\right) u_{i}\left(t^{n+1}\right)-A\left(u^{n+1}\right) u\left(u^{n+1}\right)\right\| \leq \varepsilon
$$

with sufficient iterations $i=\{1,3, \cdots, 2 m+1\}$.

Remark 8. The linearization with the fix-point scheme can be used for smooth or weak nonlinear operators, otherwise we loose the convergence behavior, while we did not converge to the local fix-point, see [21].

\section{Experiments}

In the following experiments, first we deal with the decoupled equations, means Maxwell and transport equations, to verify our methods.

In the third experiment, we consider a simple PE-CVD process and concentrate on the coupled transport and Maxwell equation.

\subsection{Test Experiment 1: Maxwell Equation}

The time-dependent Maxwell equations in 2D is given as:

$$
\frac{\partial H_{x}(x, y)}{\partial t}=-\frac{\partial E_{z}}{\partial y},(x, y, t) \in \Omega \times(0, T),
$$

$$
\begin{aligned}
& \frac{\partial H_{y}(x, y)}{\partial t}=\frac{\partial E_{z}}{\partial x},(x, y, t) \in \Omega \times(0, T), \\
& \frac{\partial E_{z}(x, y)}{\partial t}=\frac{1}{\varepsilon}\left(\frac{\partial H_{y}}{\partial x}-\frac{\partial H_{x}}{\partial y}\right)-J_{\text {source }}, \\
& (x, y, t) \in \Omega \times(0, T),
\end{aligned}
$$

where $J_{\text {source }}(x, y)=\sin (t)$.

We have to implement the outflow condition, via the underlying discretization method (we assume finite difference methods), means how many concentration is flowing via the time-step $\Delta t$ to the cell with the spatial step $\Delta x$ :

The relative spatial step is given as

$$
\sqrt{1 / \varepsilon} \Delta t=\Delta x_{\text {relativ }}
$$

The percentage of the outflow is given as:

$$
\begin{gathered}
\frac{\Delta x_{\text {relativ }}}{\Delta x}=\text { rel } \\
E_{z, \text { out }}=r e l E_{z},(x, y) \in \partial \Omega
\end{gathered}
$$

The same is also given for the $H_{x}, H_{y}$.

Here we apply the FDTD method of Yee's algorithm.

For spatial and time discretization it is important to balance such schemes.

We assume to have finite difference schemes in time and space.

Therefore the CFL (Courant Friedrichs Levy) condition is important to balance the schemes:

While we are dealing with wave-equations:

$$
\sqrt{\varepsilon} \Delta x \geq \Delta t
$$

where $\Delta x, \Delta t$ are the spatial and time steps.

To control the electric field $E_{z}(x, y)$, we have the following line source:

where

$$
\begin{aligned}
& J_{\text {source }}(x, y)=\sin (t) \\
& x=0, y \in(0,100)
\end{aligned}
$$

The control of the particle transport is given by the electric field in Figure 1. The electric and transport situation is given with cut of the three dimensional model in Figure 2.

In the following we have the line sources with the results given in Figure 3:

Remark 9. We consider the Maxwell equation, that models a periodic electric field in the reactor. We apply Yee's algorithm to obtain at least a second order scheme in time and space. Based on the slower time-scales of the Maxwell equations, which is less stiff than the transport equations, we have sufficient accuracy in the full coupled system. A higher order discretization scheme is necessary for the transport part. 


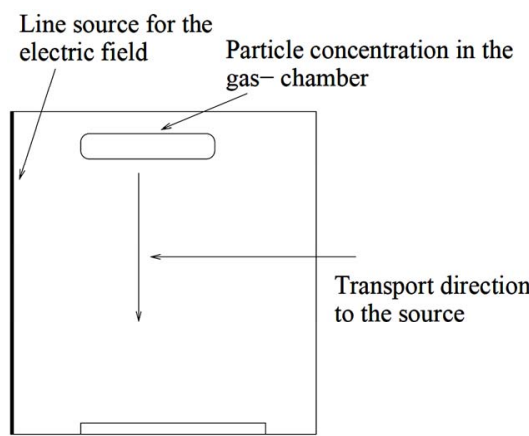

Target or source to be deposited

Figure 1. Electric field in the apparatus.

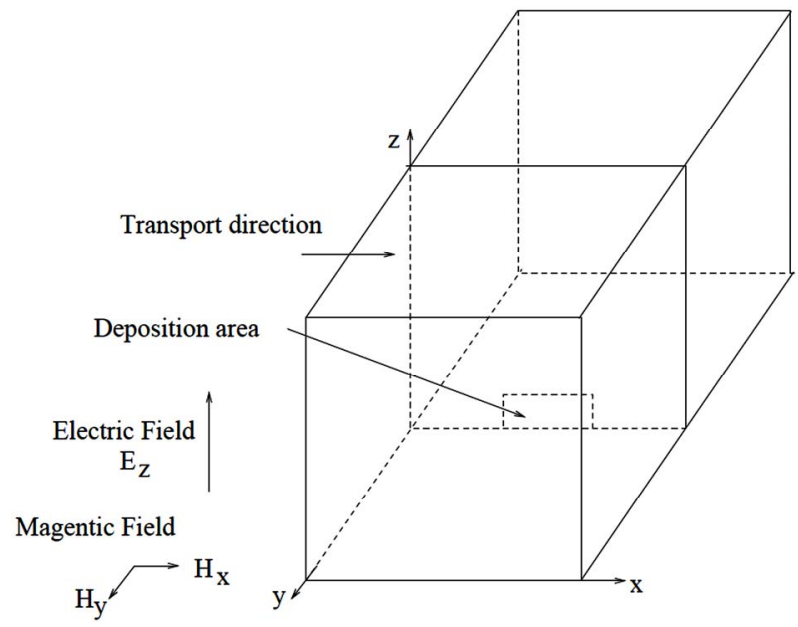

Algorithm 4.4

1) Initialize Convection-Diffusion equation, till $t_{\text {start }}$.

2) Solve Electric Field equation with $t_{s}$ tart, we obtain $E_{z}(x, y)$ for $t_{\text {start }}$.

3) Solve Convection Diffusion equation with $t_{\text {start }}+\Delta t$ and use $E_{z}(x, y)$ for $t_{\text {start }}$ for the unknown.

4) Do $t_{\text {start }}=t_{\text {start }}+\Delta t$ and go to 2.) till $t_{\text {start }}=t_{\text {end }}$

Figure 2. Electric field in the apparatus.

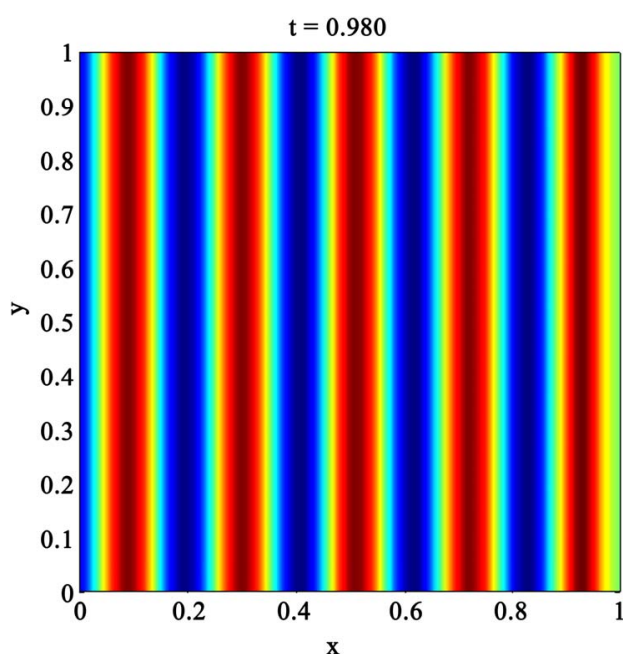

Figure 3. Line source of the Electric field in the apparatus.

\subsection{Test experiment 2: Convection-Diffusion Equation}

We deal with the 2-dimensional advection-diffusion equation and periodic boundary conditions

$$
\begin{aligned}
& \partial_{t} u=-v \nabla u+D \Delta u, \\
& \quad=-v_{x} \frac{\partial u}{\partial x}-v_{y} \frac{\partial u}{\partial y}+D \frac{\partial^{2} u}{\partial x^{2}}+D \frac{\partial^{2} u}{\partial y^{2}}, \\
& u\left(x, t_{0}\right)=u_{0}(x),
\end{aligned}
$$

with the parameters

$$
\begin{aligned}
& v_{x}=v_{y}=1 \\
& D=0.01 \\
& t_{0}=0.25 .
\end{aligned}
$$

The given advection-diffusion problem has an analytical solution

$$
u_{a}(x, t)=\frac{1}{t} \exp \left(\frac{-(x-v t)^{2}}{4 D t}\right)
$$

which we will use as a convenient initial function:

$$
u\left(x, t_{0}\right)=u_{a}\left(x, t_{0}\right)
$$

We apply dimensional splitting to our problem

$$
\frac{\partial u}{\partial t}=A_{x} u+A_{y} u
$$

where

$$
A_{x}=-v_{x} \frac{\partial u}{\partial x}+D \frac{\partial^{2} u}{\partial x^{2}} .
$$

We use a 1st order upwind scheme for $\partial / \partial x$ and a 2nd order central difference scheme for $\partial^{2} / \partial x^{2}$. By introducing the artificial diffusion constant $D_{x}=D-$ $\left(v_{x} \Delta x / 2\right)$ we achieve a 2 nd order finite difference scheme

$$
\begin{aligned}
L_{x} u(x)= & -v_{x} \frac{u(x)-u(x-\Delta x)}{\Delta x} \\
& +D_{x} \frac{u(x+\Delta x)-2 u(x)+u(x-\Delta x)}{\Delta x^{2}} .
\end{aligned}
$$

because the new diffusion constant eliminates the first order error (i.e. the numerical viscosity) of the Taylor expansion of the upwind scheme. $L_{y} u$ is derived in the same way.

We apply a BDF5 method to gain 5th order accuracy in time. For simplifications, we note that the dependencies of $u(x, t)$ are suppressed as $u(t)$.

$$
\begin{aligned}
L_{t} u(t)= & \frac{1}{\Delta t}\left(\frac{137}{60} u(t+\Delta t)-5 u(t)+5 u(t-\Delta t)\right. \\
& \left.-\frac{10}{3} u(t-2 \Delta t)+\frac{5}{4} u(t-3 \Delta t)-\frac{1}{5} u(t-4 \Delta t)\right) .
\end{aligned}
$$


To compare the four methods we have the following general setting. Let $\Omega=[0,1] \times[0,1] \times[0,1]$, the unit cube. There we set up the initial concentration

$$
\begin{aligned}
& u_{t_{0}}(x)=2 \exp \left(\frac{\left.-(x-a)^{2}\right)}{0.02}\right) \forall x \in \Omega \\
& \text { with } a=(0.5,0.5,0.5)^{\mathrm{T}}
\end{aligned}
$$

which is just the analytical solution

$$
u_{a}(x, t)=\frac{1}{t} \exp \left(\frac{-(x-v t)^{2}}{4 D t}\right)
$$

with $v=1$ and $D=0.01$ at $t=t_{0}=0.25$ on $\Omega$.

During the following experiments we will set $v=0$ and consider an equidistant lattice of $N^{3}$ points ( $\Delta x=$ $\Delta y=\Delta z=1 /(N-1))$.

The result is shown within the following Figures 4 and 5:

Remark 10. We consider the transport equation, that models the mass transport of the ionized species from the lower-left to the middle of the reactor. We use higher order time and spatial discretization schemes to obtain higher order solutions. Such methods, we can apply with larger time and spatial steps and obtain sufficient accurate results.

Based on the fast time-scales of the transport equations, which is stiffer than the Maxwell equation, we can balance the larger time-steps with sufficient accurate solution of the transport regime in the coupled system.

\subsection{Test Experiment 3: Coupling \\ Convection-Diffusion and \\ Electric Field Equations \\ (Weak Coupling)}

Here, we consider a simple PE-CVD process, that an underlying mass transport of a gaseous species is influenced by an electric field, see [1,3].

For transport in a plasma environment, we assume a homogeneous medium and that the influence of the electric field can be simulated by a coupled transport and Maxwell equation, see citelieb05.

For simplifications, we deal with the 2-dimensional advection-diffusion equation and electric field equation:

$$
\begin{gathered}
\partial_{t} u=-v_{x}\left(E_{z}(x, y)\right) \frac{\partial u}{\partial x}-v_{y} \frac{\partial u}{\partial y} \\
+D \frac{\partial^{2} u}{\partial x^{2}}+D \frac{\partial^{2} u}{\partial y^{2}}, \\
u\left(x, y, t_{0}\right)=u_{0}(x, y),
\end{gathered}
$$

$$
\begin{aligned}
& \frac{\partial H_{x}(x, y)}{\partial t}=-\frac{\partial E_{z}}{\partial y},(x, y, t) \in \Omega \times(0, T), \\
& \frac{\partial H_{y}(x, y)}{\partial t}=\frac{\partial E_{z}}{\partial x},(x, y, t) \in \Omega \times(0, T), \\
& \frac{\partial E_{z}(x, y)}{\partial t}=\frac{1}{\varepsilon}\left(\frac{\partial H_{y}}{\partial x}-\frac{\partial H_{x}}{\partial y}\right)-J_{\text {source }}, \\
& (x, y, t) \in \Omega \times(0, T),
\end{aligned}
$$

The advection-diffusion problem has an analytical solution at the beginning for $t_{0} \in\left(0, t_{\text {start }}\right)$

$$
u_{a}(x, t)=\frac{1}{t} \exp \left(\frac{-(x-v t)^{2}}{4 D t}\right)
$$

which we will use as a convenient initial function:

$$
u\left(x, t_{0}\right)=u_{a}\left(x, t_{0}\right)
$$

Further the function:

$$
\begin{aligned}
& v_{x}\left(E_{z}(x, y)\right)=1 \text { for } t \in\left(0, t_{\text {start }}\right) \\
& v_{x}\left(E_{z}(x, y)\right)=\alpha E_{z}(x, y) \text { for } t \geq t_{\text {start }}
\end{aligned}
$$

where $\alpha=0.001, t_{\text {start }}=10.0$.

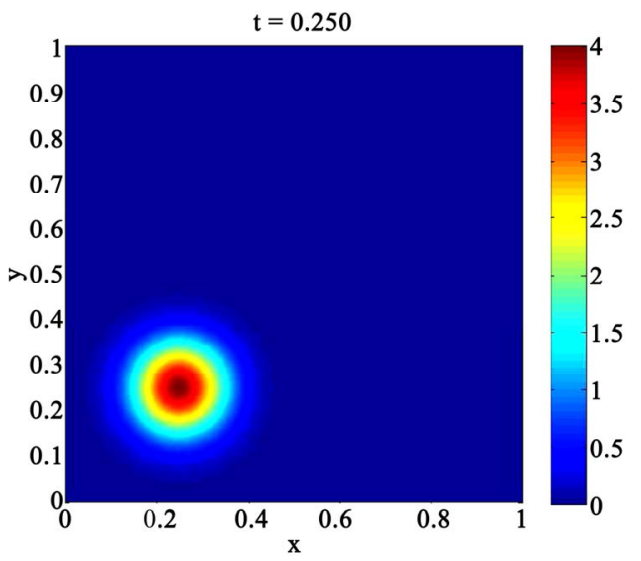

Figure 4. Initial gaseous concentration at $\boldsymbol{t}=\mathbf{0 . 2 5}$.

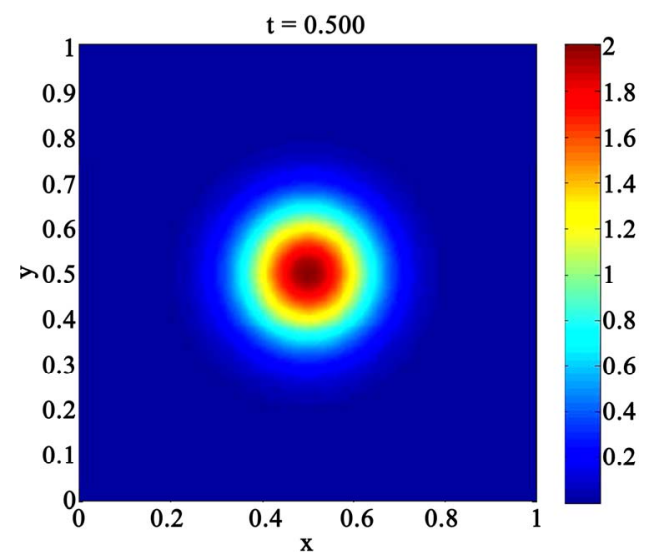

Figure 5. Gaseous concentration at $\boldsymbol{t}=\mathbf{0 . 5}$. 
Both equations have the same domain $\Omega=[0,1] \times$ $[0,1]$.

Numerically we solve the equation, as in the following algorithm 5.3:

The following figures show the developing of the concentration under the influence of the electric field, we deal with a normalized time scale in $[\mathrm{sec}]$. Further we have $\alpha=0.07, t_{\text {start }}=0.5$ and $v_{y}=0$ for $t \geq t_{\text {start }}$.

The results are given in Figure 6 - 11.

Remark 11. Based on the transport of the ionized species from the lower-left to the middle of the reactor, we see and influence of the species. The former circular concentration is spread out to a diffusive ellipse. Here, we can control the species in the reactor with a electric field. Numerically, it is important to deal with the different time- and spatial scales of the underlying transport and Maxwell equation. Via iterative splitting, we could couple the two equations systems together and reduce the numerical errors with additional iterative steps.

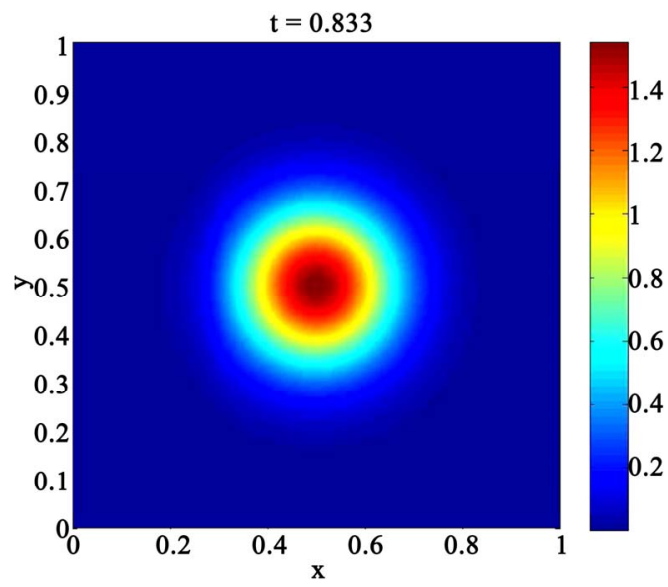

Figure 6. Gaseous concentration after $\boldsymbol{t}=\mathbf{0 . 8 3 3}$.

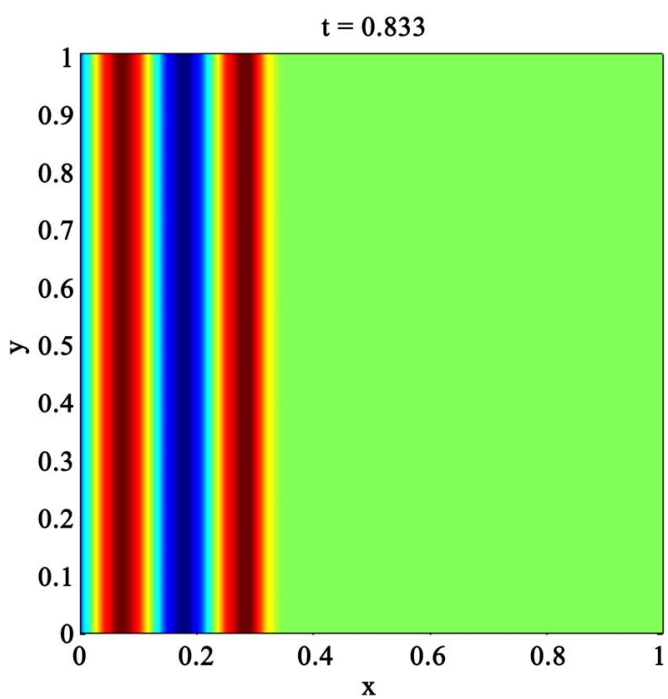

Figure 7. Electric field after $\boldsymbol{t}=\mathbf{0 . 8 3 3}$.

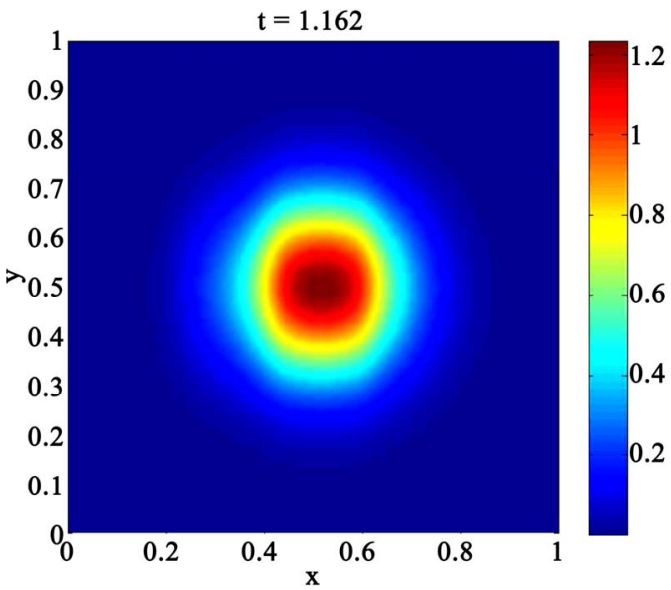

Figure 8. Gaseous concentration after $t=1.162$ with a first influence of the electric field.

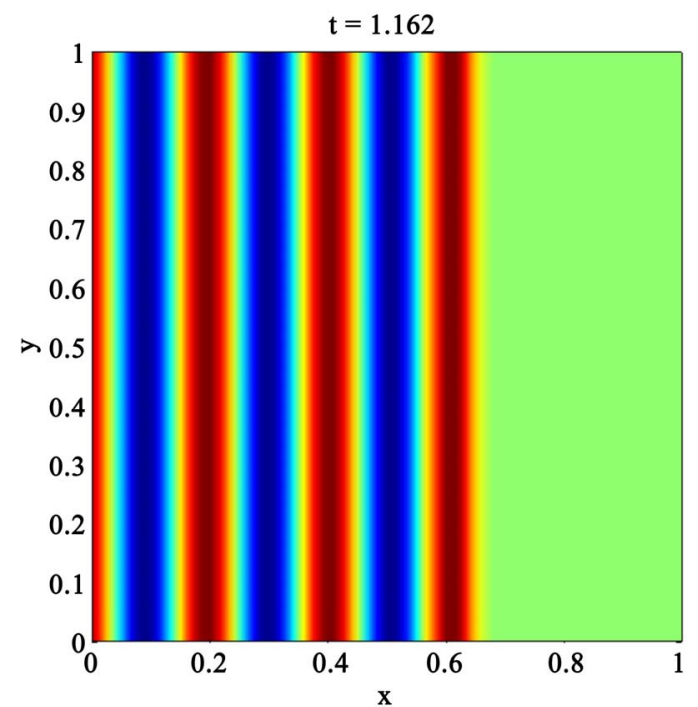

Figure 9. Electric field after $\boldsymbol{t}=\mathbf{1 . 1 6 2}$.

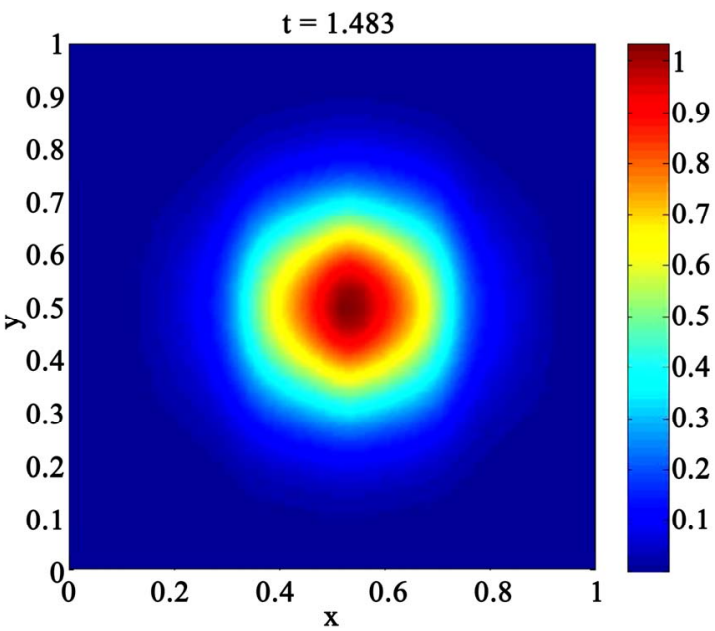

Figure 10. Gaseous concentration after $t=1.483$ with a first influence of the electric field. 


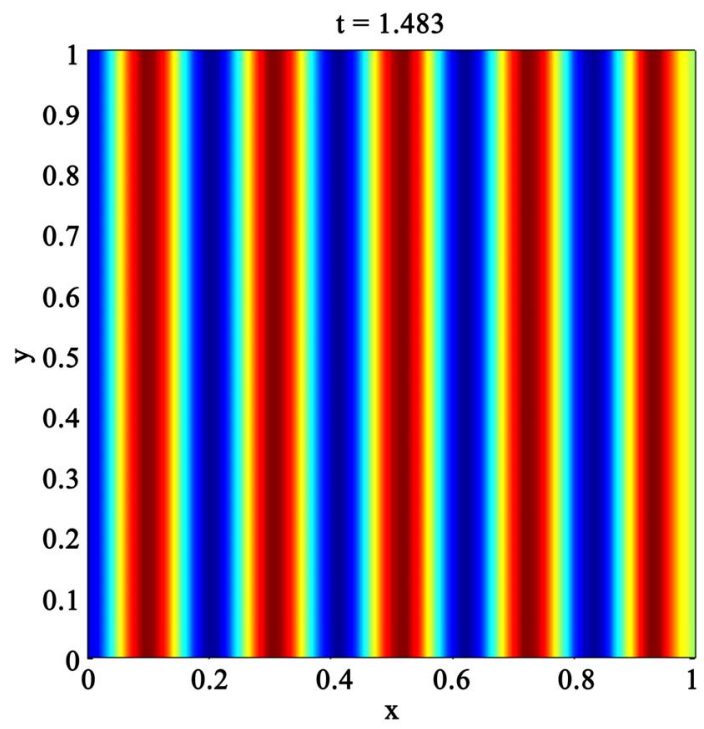

Figure 11. Electric field after $t=1.483$.

\section{Conclusions}

We present a coupled model based on Maxwell and Transport equations, that can be applied for simplified transport model for an ionized gaseous species in a PECVD reator. Based the different scale models, we have included the optimal discretization methods for each separate equation. Splitting methods are used to couple the separate equations together. Further, we discussed the splitting analysis. Numerical examples are presented to discuss the influence of decoupled and coupled systems. In future, we will analyze the validity of the models with physical experiments.

\section{References}

[1] M. Ohring, "Materials Science of Thin Films," 2nd Edition, Academic Press, San Diego, New York, Boston, London, 2002.

[2] J. Geiser and M. Arab, "Simulation of a Chemical Vapor Deposition: Mobile and Immobile Zones and Homogeneous Layers," Journal of Porous Media, Begell House Inc., Redding, 2009, Vol. 1, No. 2, pp. 123-143.

[3] L. Rudniak, "Numerical Simulation of Chemical Vapour Deposition Process in Electric Field," Computers \& Chemical Engineering, Vol. 22, Supplement 1, 1998, pp. 755-758.

[4] J. Van Roey, J. van der Donk and P. E. Lagasse, "Beam-Propagation Method: Analysis and Assessment," Journal of the Optical Society of America, Vol. 71, No. 7, 1981, pp. 803-810. doi:10.1364/JOSA.71.000803

[5] M. D. Feit and J. A. Fleck Jr., "Analysis of Rib Waveguides and Couplers by the Propagating Beam Method," Journal of the Optical Society of America A, Vol. 7, No. 1, 1990, pp. 73-79.
doi:10.1364/JOSAA.7.000073

[6] M. D. Feit and J. A. Fleck Jr., "Light Propagation in Graded-Index Optical Fibers," OSA Applied Optics, Vol. 17, No. 24, 1978, pp. 3990- 3998. doi:10.1364/AO.17.003990

[7] L. Thylen, E. M. Wright, G. I. Stegeman, C. T. Seaton and J. V. Moloney, "Beam-Propagation Method Analysis of a Nonlinear Directional Coupler," OSA Optics Letters, Vol. 11, No. 11, 1986, pp. 739-741. doi:10.1364/OL.11.000739

[8] J. A. Fleck, J. R. Morris Jr. and M. D. Feit, "Time-dependent Propagation of High Energy Laser Beams through the Atmosphere," Applied Physics, Vol. 10, No. 2, 1976, pp. 129-160. doi:10.1007/BF00896333

[9] M. Lax, J. H. Batteh and G. P. Agrawai, "Channeling of Intense Electromagnetic Beams," Journal of Applied Physics, Vol. 51, No. 1, 1981, pp. 109-125. doi:10.1063/1.328442

[10] G. I. Marchuk, "Some Applications of Splitting-up Methods to the Solution of Mathematical Physics Problems," Aplikace Matematiky, Vol. 1, 1968, pp. 103-132.

[11] G. Strang, "On the Constraction and Comparion of Difference Schemes," SIAM J. Numerical Analysis, Vol. 5, No. 3, 1968, pp. 506-517. doi:10.1137/0705041

[12] K. Okamoto, "Fundamentals of Optical Waveguides," Academic Press, New York, 2005.

[13] A Taflove, "Computational Electrodynamics: The Finite Difference Time Domain Method," Arctech House Inc., 1995.

[14] J. P. Berenger, "A Perfectly Matched Layer for the Absorption of Electromagnetic Waves," J. Comp. Phys., Vol. 111, 2005, pp. 185-220.

[15] S. D. Gedney, "An Anisotropic Perfectly Matched Layer-Absorbing Medium for the Truncation of Fdtd Lattices," IEEE Tran. Ant. Prop., Vol. 44, No. 12, 1996, pp. 1630-1639.

[16] W. Sha, X. Wu, M. Chen and Z. Huang, "Application of the High-Order Symplectic Fdtd Scheme to the Curved Three-Dimensional Perfectly Conducting Objects,"

[17] T. Hirono, W. Lui, S. Seki and Y. Yoshikuni, "A ThreeDimensional Fourth-Order Finite-Difference Time-Domain Scheme Using a Symplectic Integrator Propagator,"

[18] J. Geiser, "Numerical Simulation of a Model for Transport and Reaction of Radionuclides, 2001," Proceedings of the Large Scale Scientific Computations of Engineering and Environmental Problems, Sozopol, 2001.

[19] W. Hackbusch, "Iterative Losung Groser Schwachbesetzter Gleichungssysteme," Teubner-Verlag, Stuttgart, 1993.

[20] I. Farago and J. Geiser, "Iterative Operator-Splitting Methods for Linear Problems," International Journal of Computational Science and Engineering, Vol. 3, No. 4, 2007, pp. 255-263.

[21] J. Kanney, C. Miller and C. Kelley, "Convergence of Iterative Split-Operator Approaches for Approximating 
Nonlinear Reactive Transport Problems," Advances in Water Resources, Vol. 26, 2003, pp. 247-261.

doi:10.1016/S0309-1708(02)00162-8

[22] J. Geiser, "Weighted Iterative Operator-Splitting Methods: Stability-Theory," Proceedings of the 6th International Conference, NMA 2006, Lecture Notes in Computer Science, Springer, Berlin, Vol. 4310, 2007, pp. 40-47.

[23] W. H. Hundsdorfer and J. G. Verwer, "Numerical Solution of Time-Dependent Advection-Diffusionreaction
Equations," Springer, Berlin, 2003.

[24] J. Geiser, "Iterative Operator-Splitting Methods with Higher Order Time-Integration Methods and Applications for Parabolic Partial Differential Equations," Journal of Computational and Applied Mathematics, Elsevier, Amsterdam, Vol. 217, 2008, pp. 227-242.

[25] E. Zeidler, "Nonlinear Functional Analysis and Its Applications II/B: Nonlinear Montone Operators," SpringerVerlag, Berlin-Heidelberg-New York, 1990. 